\title{
Eficacia y seguridad de la fisioterapia respiratoria en pacientes adultos con neumonía adquirida en la comunidad
}

\author{
FERNANDO SALDÍAS P.* y ORLANDO DÍAZ P.*
}

\author{
Efficacy and safety of chest physiotherapy in adult patients \\ with community-acquired pneumonia
}

Pneumonia is one of the most common health problems affecting all age groups around the world. Chest physiotherapy includes a variety of techniques, such as conventional chest physiotherapy (i.e., percussion and postural drainage), active cycle of breathing techniques, positive expiratory pressure (using devices to maintain 10 to $25 \mathrm{~cm}$ of water pressure during expiration), and osteopathic manipulation. In theory, these techniques could help keep airways open, improve secretion clearance, and increase gas exchange. Chest physiotherapy has been widely used as an adjunctive therapy for pneumonia in adults without any reliable evidence. We evaluate the evidence examining the efficacy and safety of chest physiotherapy in adult patients with community-acquired pneumonia. Results: Six randomized controlled trials have assessed four types of chest physiotherapy, namely conventional chest physiotherapy, osteopathic manipulative treatment (including paraspinal inhibition, rib raising, and diaphragmatic or soft myofascial release), active cycle of breathing techniques (including active breathing control, thoracic expansion exercises and forced expiration technique) and positive expiratory pressure, in community-acquired pneumonia hospitalized patients. None of these techniques (versus no physiotherapy or placebo therapy) reduce mortality or improve cure rate. Limited evidence indicates that osteopathic manipulative treatment (versus placebo therapy) and positive expiratory pressure (versus no physiotherapy) can slightly reduce the duration of hospital stay (by 2.0 and 1.4 days, respectively). In addition, positive expiratory pressure (versus no physiotherapy) can slightly reduce the duration of fever by 0.7 day, and osteopathic manipulative treatment (versus placebo therapy) might reduce the duration of antibiotic use by 1.93 days. No severe adverse events were reported. In summary, the available evidence is limited and chest physiotherapy should not be recommended as routine adjunctive treatment for non-complicated pneumonia in adults.

Key words: Community-acquired pneumonia, evidence-based medicine, physical therapy, physiotherapy, respiratory tract infections.

\section{Resumen}

La neumonía adquirida en la comunidad es la principal causa de muerte de origen infeccioso en la población infantil y adulta de nuestro país. La fisioterapia respiratoria (FR) ha sido ampliamente utilizada como tratamiento adyuvante en pacientes adultos con neumonía comunitaria, sin que se haya demostrado aún su eficacia clínica. En teoría, estas técnicas podrían ayudar a mantener abiertas las vías aéreas, facilitar la eliminación de secreciones bronquiales y mejorar el intercambio de gases en pacientes con neumonía. Revisamos los estudios publicados que han examinado la eficacia y seguridad de la FR en pacientes adultos con neumonía adquirida en la comunidad. Resultados: Seis ensayos clínicos controlados han evaluado cuatro modalidades de fisioterapia respiratoria en pacientes hospitalizados por neumonía comunitaria: a) La fisioterapia torácica convencional (vibración, percusión y drenaje postural); b) La manipulación osteopática (incluyendo la inhibición paraespinal, elevación costal y

* Comisión de Infecciones Respiratorias, Sociedad Chilena de Enfermedades Respiratorias. Departamento de Enfermedades Respiratorias, Facultad de Medicina, Pontificia Universidad Católica de Chile. 
la liberación diafragmática o miofascial); c) El ciclo activo de técnicas de respiración (incluyendo el control de la respiración activa, ejercicios de expansión torácica y técnicas de espiración forzada); y d) La respiración con presión espiratoria positiva. Ninguna de estas técnicas ha logrado reducir en forma significativa la mortalidad hospitalaria o ha modificado la tasa de curación. Algunos estudios sugieren que la manipulación osteopática y la respiración con presión espiratoria positiva pueden acortar la estadía hospitalaria (2,0 y 1,4 días, respectivamente). Además, la respiración con presión espiratoria positiva puede reducir la duración de la fiebre en 0,7 dias, y la manipulación osteopática puede acortar el uso de antibióticos en 1,93 días. No se han reportado eventos adversos serios. En resumen, la evidencia disponible es limitada y sugiere que la fisioterapia respiratoria no debería ser recomendada como tratamiento adyuvante de rutina en pacientes adultos con neumonía comunitaria no complicada.

Palabras clave: Neumonía adquirida en la comunidad, medicina basada en la evidencia, terapia fisica, fisioterapia, infecciones respiratorias.

\section{Introducción}

La neumonía adquirida en la comunidad es la principal causa de muerte de origen infeccioso en la población infantil y adulta de nuestro país ${ }^{1}$. De acuerdo a su gravedad, se estima que el 70 a $80 \%$ de los pacientes pueden ser manejados en el ámbito ambulatorio con bajo riesgo de complicaciones y muerte (letalidad inferior a $1 \%)^{2}$. El manejo de los enfermos considera medidas de cuidados generales (reposo, hidratación, oxígenoterapia, fisioterapia respiratoria) y el tratamiento antibiótico empírico con cobertura sobre los principales patógenos respiratorios basado en las recomendaciones de las guías clínicas ${ }^{3-5}$.

La fisioterapia respiratoria hace referencia al conjunto de técnicas físicas encaminadas a eliminar las secreciones de la vía aérea y mejorar la ventilación pulmonar ${ }^{6,7}$. Los principales objetivos de las técnicas de terapia física son mantener la permeabilidad de la vía aérea, facilitar la movilización y eliminación de secreciones bronquiales y prevenir complicaciones respiratorias agudas (ej. hipoxemia, obstrucción bronquial, atelectasias, infección) ${ }^{7}$. La evidencia científica que justificaría su empleo en pacientes con infecciones del tracto respiratorio inferior es insuficiente, aunque la práctica habitual perpetúa su uso. Las principales limitaciones en el diseño de los estudios son la imposibilidad de mantener ciegos a pacientes y terapeutas, la falta de consenso en la técnica estándar con qué comparar las nuevas técnicas y la escasa precisión de las variables empleadas en la evaluación de los resultados.

\section{Caso clínico}

Hombre de 65 años, fumador de 20 paq/año, fuma 4-5 cig/día, portador de hipertensión arterial crónica y diabetes mellitus tipo 2 , es admitido en la sala de medicina con una neumonía adquirida en la comunidad que afecta los lóbulos medio e inferior derecho (Figura 1). El médico tratante se comunica con el equipo de kinesiología y recomienda el empleo de técnicas de fisioterapia respiratoria para tratar de reducir la duración de su estancia en el hospital, riesgo de complicaciones y muerte. Nos preguntamos si existe evidencia que apoye el uso de la fisioterapia en este caso.

\section{Pregunta clínica}

¿En el paciente adulto con neumonía adquirida en la comunidad [del paciente], la fisioterapia torácica [la intervención] permite acortar la estadía en el hospital, reducir las complicaciones y el riesgo de muerte [el resultado]?

Pacientes: Adultos inmunocompetentes atendidos en el ámbito ambulatorio y hospitalario con neumonía adquirida en la comunidad.

Intervención: Fisioterapia respiratoria (percusión, vibración, drenaje postural, respiración con presión positiva intermitente, tos asistida y ayuda externa con la respiración) en pacientes adultos con neumonía adquirida en la comunidad.

Resultado: Duración de los síntomas y signos, resolución de los infiltrados radiográficos, mejoría clínica, estancia hospitalaria, complicaciones y letalidad.

\section{Revisión de la evidencia}

Los estudios clínicos ${ }^{8-16}$ que han examinado la utilidad de la fisioterapia respiratoria en pacientes con neumonía comunitaria no han logrado demostrar que sea eficaz en los siguientes aspectos: a) Velocidad de resolución de los síntomas y signos clínicos; b) Velocidad de resolución de los infiltrados radiográficos; c) Mejoría clínica subjetiva; d) Estadía en el hospital y e) Letalidad 


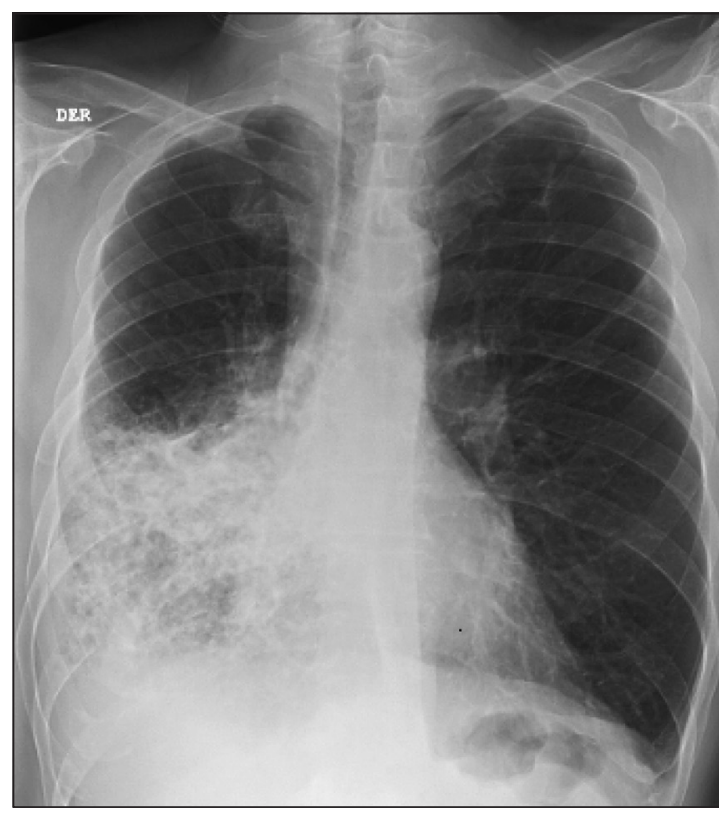

Figura 1. Radiografía de tórax PA. Hombre de 65 años, fumador, con foco de condensación no homogéneo en el lóbulo medio y lóbulo inferior derecho.

en el hospital. Los estudios clínicos controlados han sido escasos ${ }^{8-16}$ (Tabla 1), con diseño metodológico variable (estudios controlados randomizados, estudios de cohorte), incluyen población pediátrica ${ }^{8,10,16}$ y adulta ${ }^{9,11,12-15}$, han examinado diferentes modalidades de fisioterapia respiratoria: percusión, vibración, drenaje postural, respiración con presión positiva intermitente y ayuda externa con la respiración.

La fisioterapia respiratoria incluye una variedad de técnicas, como la fisioterapia torácica convencional (es decir, percusión, vibración y drenaje postural), diferentes técnicas de respiración activa, la respiración con presión espiratoria positiva (el uso de dispositivos para mantener una presión de 10 a $25 \mathrm{~cm}$ de agua durante la espiración), y la manipulación osteopática ${ }^{7}$. En teoría, estas técnicas podrían ayudar a mantener abiertas las vías aéreas, mejorar la depuración de secreciones bronquiales y el intercambio de gases.

En los pacientes con neumonía, sólo se han estudiado las técnicas tradicionales para facilitar la eliminación de las secreciones de las vías respiratorias (ejercicios de respiración, drenaje postural, percusión y vibración $)^{17}$. Cabe señalar que estos estudios se realizaron en pacientes con neumonía no complicada y no se incluyeron pacientes con enfermedad pulmonar obstructiva crónica (EPOC) u otras afecciones respiratorias crónicas. Además, en la práctica clínica habitual no se recomienda el empleo de estas técnicas en pa- cientes con neumonía, a menos que la producción de esputo sea abundante o exista dificultad para expectorar, poniendo el énfasis en la movilización precoz, restauración de la función y corrección de la insuficiencia respiratoria o ventilatoria ${ }^{6}$.

En una revisión sistemática, Yang y $\operatorname{cols}^{17}$, identificaron seis estudios de fisioterapia respiratoria en pacientes adultos con neumonía ( $n=434 ; 215$ hombres y 219 mujeres; rango de edad: 15-94 años; casos: 211 y controles: 223$)^{8-16}$. No se incluyeron siete estudios que inicialmente parecían cumplir con los criterios de inclusión debido a que cinco de ellos fueron publicados en ruso $^{18-22}$, uno era inédito ${ }^{23}$, y uno se publicó en 1947 y no estaba disponible para los autores ${ }^{24}$. Todos los estudios eran randomizados, aunque en ninguno de ellos se describe la modalidad de randomización, en tres estudios los participantes eran ciegos ${ }^{11,14,15}$, en dos eran ciegos los evaluadores ${ }^{14,15}$, y en tres hubo ocultamiento de la asignación $\mathrm{n}^{9,11,13}$. Todos los pacientes recibieron el tratamiento habitual (reposo, hidratación, oxigenoterapia y antibióticos) recomendado en las guías clínicas. La fisioterapia torácica se comparó con la terapia de placebo en dos de los estudios $^{14,15}$ y con el tratamiento convencional en cuatro de los estudios ${ }^{9,11-13}$. Ninguna de las intervenciones demostró mejoría de la mortalidad, ${ }^{9,11-15}$ o la tasa de curación ${ }^{9,11,12,14,15}$. La manipulación osteopática, examinada en dos ensayos doble ciego controlados por simulación, con 79 pacientes, demostraron una reducción de la estancia hospitalaria en una media de dos días y la duración del tratamiento antibiótico ${ }^{14,15}$.

Los seis estudios controlados examinaron cuatro modalidades de fisioterapia respiratoria: a) La fisioterapia torácica convencional ${ }^{9,11}$; b) El tratamiento de manipulación osteopática (que incluye la inhibición paraespinal, elevación de las costillas y liberación miofascial) ${ }^{14,15}$; c) El ciclo activo de técnicas de respiración (que incluyen el control de la respiración activa, ejercicios de expansión torácica y técnicas de espiración forzada) ${ }^{12}$; y d) La respiración con presión espiratoria positiva $^{13}$ (Tabla 2). Ninguna de las modalidades de fisioterapia torácica (versus terapia habitual o placebo) demostró reducción significativa de la mortalidad en adultos hospitalizados por neumonía; tampoco aumentaron la tasa de curación y velocidad de resolución de los síntomas, signos e infiltrados radiográficos. La manipulación osteopática (versus placebo) y la respiración con presión espiratoria positiva (versus terapia habitual) redujeron la duración media de la estancia hospitalaria en 2,0 días (diferencia media (DM) $-2,0$ días, IC 95\%: $-3,5$ a -0,6) y 1,4 días (DM 


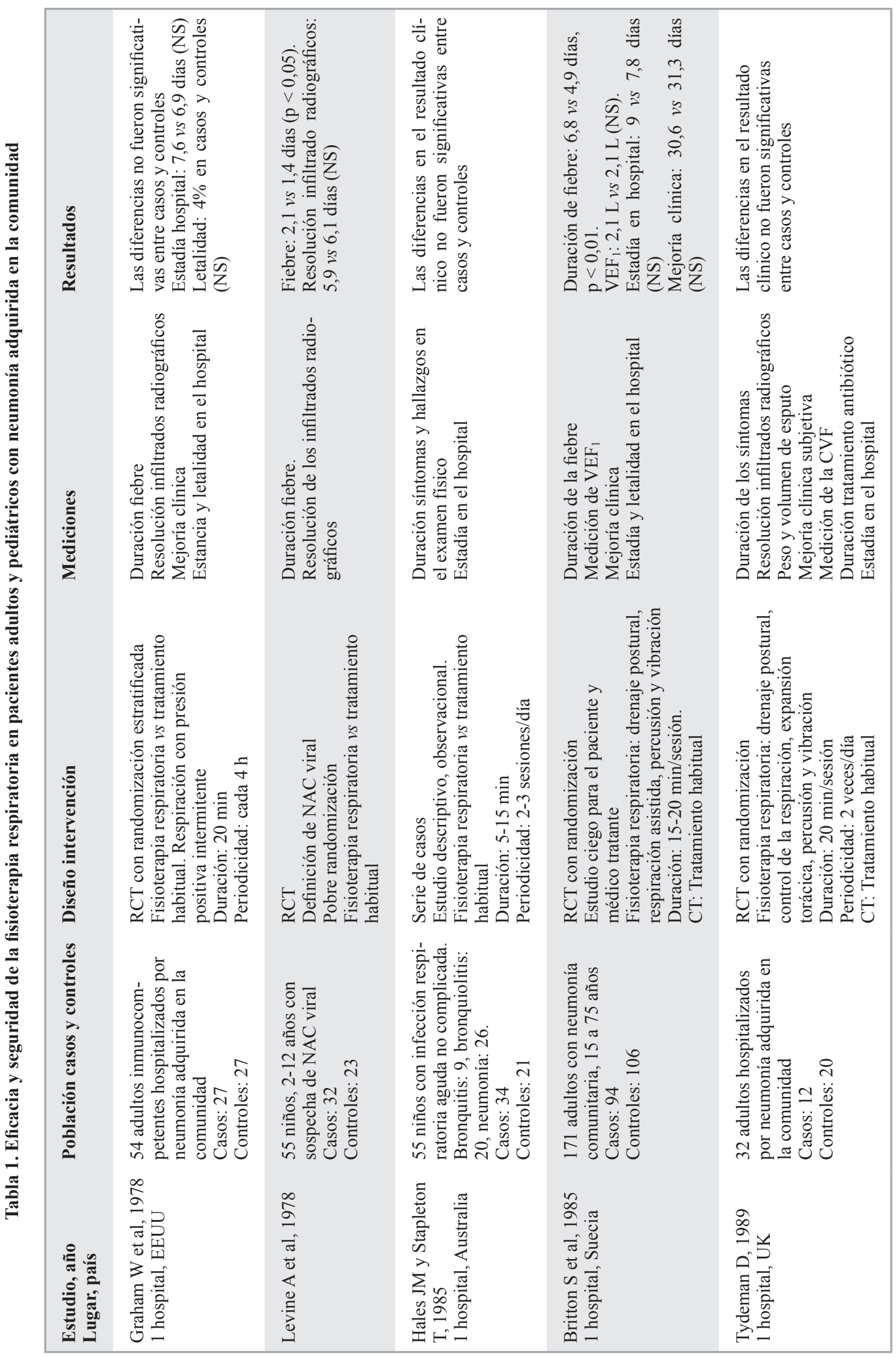




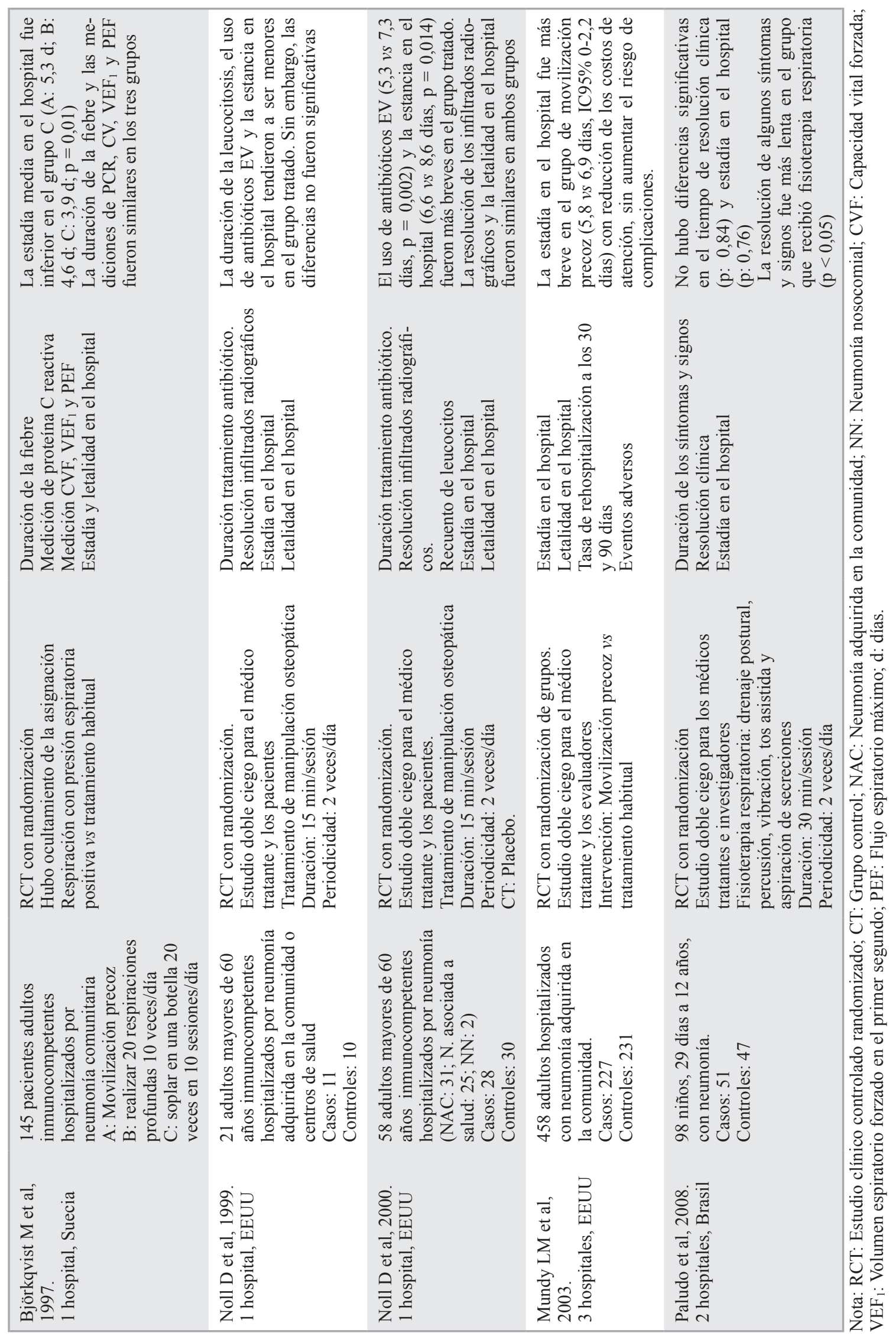


Tabla 2. Eficacia de las diferentes modalidades de fisioterapia respiratoria en pacientes adultos hospitalizados por neumonía adquirida en la comunidad

\begin{tabular}{|c|c|c|c|c|}
\hline Fisioterapia respiratoria tradicional & $\begin{array}{c}\mathbf{n} \text { de } \\
\text { estudios }\end{array}$ & $\begin{array}{c}\text { Eventos/casos } \\
\text { Media } \pm \text { DE }\end{array}$ & $\begin{array}{l}\text { Eventos/controles } \\
\quad \text { Media } \pm \mathrm{DE}\end{array}$ & $\begin{array}{l}\text { Razón de riesgo } \\
\text { Diferencia media } \\
\text { (IC95\%) }\end{array}$ \\
\hline Mortalidad en el hospital & 2 & $2 / 110$ & $2 / 115$ & $1,03(0,15-7,13)$ \\
\hline Tasa de curación & 2 & $99 / 110$ & $107 / 115$ & $0,97(0,91-1,04)$ \\
\hline Estadía en el hospital (días) & 1 & $7,6 \pm 3,6$ & $6,9 \pm 4,1$ & DM $0,70(-1,3-2,7)$ \\
\hline Duración de la fiebre (días) & 1 & $2,9 \pm 2,0$ & $2,5 \pm 3,1$ & DM $0,40(-1,0-1,8)$ \\
\hline Resolución de los infiltrados radiográficos & 1 & $17 / 27$ & $20 / 27$ & $0,85(0,59-1,22)$ \\
\hline $\begin{array}{l}\text { Ciclo activo de técnicas } \\
\text { de respiración }\end{array}$ & $\begin{array}{c}\text { n de } \\
\text { estudios }\end{array}$ & $\begin{array}{c}\text { Eventos/casos } \\
\text { Media } \pm \text { DE }\end{array}$ & $\begin{array}{l}\text { Eventos/controles } \\
\quad \text { Media } \pm \mathrm{DE}\end{array}$ & $\begin{array}{l}\text { Razón de riesgo } \\
\text { Diferencia media } \\
\text { (IC95\%) }\end{array}$ \\
\hline Mortalidad en el hospital & 1 & $0 / 12$ & $0 / 20$ & No hubo decesos \\
\hline Tasa de curación & 1 & $5 / 12$ & $14 / 20$ & $0,60(0,29-1,23)$ \\
\hline Estadía en el hospital (días) & 1 & $6,6 \pm 3,2$ & $5,3 \pm 2,2$ & DM 1,40 $(-0,6-3,4)$ \\
\hline Resolución de los infiltrados radiográficos & 1 & $5 / 12$ & $14 / 20$ & $0,60(0,29-1,23)$ \\
\hline Duración del tratamiento antibiótico & 1 & $15,1 \pm 6,7$ & $15,0 \pm 5,5$ & DM 0,15 $(-4,3-4,6)$ \\
\hline $\begin{array}{l}\text { Técnicas de manipulación } \\
\text { osteopática }\end{array}$ & $\begin{array}{l}\text { n de } \\
\text { estudios }\end{array}$ & $\begin{array}{c}\text { Eventos/casos } \\
\text { Media } \pm \text { DE }\end{array}$ & $\begin{array}{l}\text { Eventos/controles } \\
\quad \text { Media } \pm \mathrm{DE}\end{array}$ & $\begin{array}{l}\text { Razón de riesgo } \\
\text { Diferencia media } \\
\text { (IC95\%) }\end{array}$ \\
\hline Mortalidad en el hospital & 2 & $1 / 39$ & $5 / 40$ & $0,27(0,05-1,57)$ \\
\hline Tasa de curación & 2 & $23 / 39$ & $15 / 40$ & $1,54(0,97-2,46)$ \\
\hline Estadía en el hospital (días) & 1 & $6,6 \pm 2,9$ & $8,6 \pm 2,9$ & DM $-2,0(-3,4$ a $-0,5)$ \\
\hline Resolución de los infiltrados radiográficos & 2 & $21 / 36$ & $19 / 39$ & $1,16(0,77-1,73)$ \\
\hline Duración del tratamiento antibiótico (días) & 1 & $6,1 \pm 2,3$ & $8,1 \pm 2,5$ & DM $-2,0(-3,2$ a $-0,7)$ \\
\hline $\begin{array}{l}\text { Respiración con presión positiva } \\
\text { espiratoria }\end{array}$ & $\begin{array}{l}\text { n de } \\
\text { estudios }\end{array}$ & $\begin{array}{c}\text { Eventos/casos } \\
\text { Media } \pm \text { DE }\end{array}$ & $\begin{array}{l}\text { Eventos/controles } \\
\quad \text { Media } \pm \text { DE }\end{array}$ & $\begin{array}{l}\text { Razón de riesgo } \\
\text { Diferencia media } \\
\text { (IC95\%) }\end{array}$ \\
\hline Mortalidad en el hospital & 1 & $0 / 50$ & $0 / 48$ & No hubo decesos \\
\hline Estadía en el hospital (días) & 1 & $3,9 \pm 2,9$ & $5,3 \pm 3,9$ & DM $-1,4 \quad(-2,7$ a $-0,03)$ \\
\hline Duración de la fiebre (días) & 1 & $1,6 \pm 1,0$ & $2,3 \pm 2,1$ & DM $-0,70(-1,3$ a $-0,04)$ \\
\hline
\end{tabular}

$-1,4$ días, IC 95\%: -2,8 a -0,0), respectivamente. La respiración con presión espiratoria positiva (versus terapia habitual) redujo la duración de la fiebre en menos de un día (DM -0,7 días, IC 95\%: -1,4 a -0,0). El tratamiento de manipulación osteopática (versus placebo) redujo la duración de la administración de antibióticos intravenoso (DM -2,1 días, IC 95\%: -3,4 a -0,9) y la duración total del tratamiento antibiótico (DM -1,9 días, IC 95\%: -3,1 a -0,7). Las principales limitaciones de esta revisión fueron el pequeño tamaño muestral de los estudios que examinaron el tratamiento de manipulación osteopática y los seis estudios publicados que parecían cumplir los criterios de inclusión y que están a la espera de clasificación ${ }^{18-22,24}$.

En un estudio controlado, Mundy y cols, evaluaron la utilidad de la movilización precoz comparado con la terapia estándar en 458 pacientes adultos hospitalizados por neumonía adquirida en la comunidad ${ }^{25}$. La movilización precoz consistía en sentarse fuera de la cama por lo menos $20 \mathrm{~min}$ durante las primeras $24 \mathrm{~h}$ del ingreso hospitalario, con aumento progresivo de la movilidad en los días posteriores. Los autores reportaron una reducción significativa de la estancia hospitalaria en el grupo de movilización temprana comparado con el grupo control (me- 
dia: 5,8 vs 6,9 días; diferencia absoluta ajustada: 1,1 días; IC95\% 0-2,2 días). La mortalidad (9,7\% vs $8,7 \%$ ) y la tasa de readmisiones hospitalarias en el seguimiento a 90 días fueron similares en ambos grupos. En conclusión, la movilización precoz de los pacientes hospitalizados por neumonía comunitaria permitió acortar la estancia hospitalaria y reducir los costos de la atención de salud, sin aumentar significativamente el riesgo de eventos adversos.

En la actualidad no disponemos de información clínica fehaciente que permita avalar la utilidad de la fisioterapia respiratoria en pacientes pediátricos con neumonía ${ }^{26}$, los estudios clínicos han sido escasos y no han demostrado beneficios en términos de resolución de los síntomas, signos y anomalías radiológicas, estancia hospitalaria y letalidad (Tabla 1). Se han realizado tres estudios que examinaron el papel de la fisioterapia respiratoria en pacientes pediátricos con neumonía ${ }^{8,10,16}$. En un ensayo clínico controlado, 55 pacientes pediátricos con neumonía primaria y sin patología respiratoria subyacente fueron asignados a recibir fisioterapia respiratoria o tratamiento convencional ${ }^{8}$. La fisioterapia torácica no demostró beneficio clínico en términos de resolución de los síntomas y alteraciones radiográficas. Esto llevó a una editoria ${ }^{27}$ que sugiere que la fisioterapia respiratoria pudiera ser perjudicial en algunos pacientes, especialmente aquellos que no producen expectoración excesiva.

En general, se acepta que la aplicación de técnicas manuales en pacientes con condensación pulmonar no tendría beneficio clínico; sin embargo, podría ser útil el posicionamiento de los pacientes para mejorar la relación ventilación/ perfusión ${ }^{28}$. Una vez que la fase de consolidación pulmonar se comienza a resolver, las técnicas de fisioterapia torácica podrían tener algún beneficio en la movilización y eliminación de las secreciones, especialmente en los niños y adultos con debilidad muscular o que no cooperan.

Con el propósito de optimizar los costos de la atención de salud, Guessous y cols, examinaron la implementación de una guía clínica para la prescripción de fisioterapia respiratoria en pacientes adultos inmunocompetentes hospitalizados por neumonía comunitaria en la sala de cuidados generale ${ }^{29}$. Los autores lograron reducir en un $30 \%$ la prescripción de fisioterapia respiratoria y más del $50 \%$ de los costos asociados a su prescripción, siendo recomendada en pacientes con enfermedad respiratoria crónica severa (EPOC) o enfermedad neuromuscular e hipersecreción bronquial, sin aumentar significativamente el riesgo de eventos adversos: la letalidad en el hospital y la tasa de rehospitalización en el siguiente año.

Las revisiones sistemáticas sugieren que la fisioterapia respiratoria convencional y otras modalidades como la respiración con presión espiratoria positiva y los ejercicios respiratorios activos facilitarían la eliminación de secreciones bronquiales en pacientes portadores de daño pulmonar crónico (EPOC, bronquiectasias, fibrosis quística), especialmente en aquellos con hipersecreción bronquial ${ }^{6,730-35}$.

En nuestra revisión no encontramos estudios clínicos controlados que hayan examinado la eficacia y seguridad de la fisioterapia respiratoria en pacientes adultos con neumonía adquirida en la comunidad manejados en el ámbito ambulatorio (categoría de bajo riesgo de complicaciones y muerte) o en la unidad de cuidados intensivos con criterios de neumonía grave.

Los reportes de seguridad han confirmado que las técnicas de fisioterapia respiratoria son bien toleradas por los enfermos y con bajo perfil de riesgo. Se han reportado episodios de desoxigenación transitoria ${ }^{36,37}$, aumento de la demanda metabólica $^{38,39}$, obstrucción bronquial difusa ${ }^{40,41}$ e incluso fracturas costales ${ }^{42}$ después de la fisioterapia respiratoria, especialmente en pacientes inestables con neumonía grave y portadores de enfermedades pulmonares obstructivas crónicas.

La fisioterapia respiratoria puede ser indicada a pacientes con neumonía adquirida en la comunidad, especialmente aquellos con hipersecreción bronquial (más de $30 \mathrm{ml} /$ día) y enfermedad respiratoria crónica (EPOC, bronquiectasias, secuelas de TBC pulmonar) ${ }^{43}$. No se encontraron ensayos clínicos que apoyaran el uso de la fisioterapia torácica de rutina para el tratamiento de pacientes adultos con neumonía comunitaria no complicada ${ }^{17}$.

\section{Efecto potencial}

En pacientes adultos con neumonía comunitaria, la fisioterapia respiratoria no ha demostrado beneficios clínicos en términos de mejoría clínica, resolución de los síntomas y signos, resolución de los infiltrados radiográficos, estadía en el hospital y letalidad. Evidencia limitada sugiere que las técnicas de manipulación osteopática y respiración con presión espiratoria positiva acortarían la estadía hospitalaria reduciendo los costos de la atención sanitaria. Las diferentes modalidades de fisioterapia respiratoria aplicadas a pacientes con neumonía comunitaria no complicada han demostrado un buen perfil de seguridad con bajo riesgo de eventos adversos. 


\section{Evaluación del costo/beneficio}

De acuerdo a la información disponible, no es posible asegurar que la fisioterapia respiratoria en pacientes adultos con neumonía adquirida en la comunidad sea costo/efectiva. Es conveniente realizar estudios clínicos randomizados, multicéntricos, para aclarar este aspecto.

\section{Recomendaciones}

a) Los pacientes adultos hospitalizados por neumonía adquirida en la comunidad no complicada deberían permanecer fuera de la cama por lo menos 20 min durante las primeras $24 \mathrm{~h}$ y aumentar progresivamente la movilidad en los días siguientes a su hospitalización (recomendación moderada sustentada en evidencia de buena calidad).

b) En los pacientes hospitalizados por neumonía comunitaria no complicada no se recomienda el empleo de las técnicas de fisioterapia respiratoria tradicionales de forma rutinaria (recomendación moderada sustentada en evidencia de regular calidad).

c) Tenga en cuenta las técnicas de fisioterapia respiratoria en los pacientes con hipersecreción bronquial y dificultad para expectorar o en el caso de una enfermedad pulmonar pre-existente (recomendación moderada sustentada en evidencia de buena calidad).

d) En los pacientes adultos hospitalizados con neumonía comunitaria no complicada, el uso regular de las técnicas de respiración con presión espiratoria positiva deberían ser consideradas (recomendación moderada sustentada en evidencia de regular calidad).

e) Los pacientes ingresados al hospital con neumonía comunitaria no complicada no deberían ser tratados con las técnicas de fisioterapia respiratoria tradicionales en combinación con las técnicas de presión positiva intermitente (recomendación moderada sustentada en evidencia de regular calidad).

En resumen, es recomendable realizar nuevos ensayos clínicos controlados para poder precisar el papel de la fisioterapia respiratoria en pacientes adultos con neumonía adquirida en la comunidad manejados en el ámbito ambulatorio y hospitalario (sala de cuidados generales y unidad de cuidados intensivos). Los siguientes puntos claves deberían ser considerados en los estudios: a) El tamaño apropiado de la muestra con el poder estadístico necesario para poder detectar diferencias clínicamente relevantes; b) La estandarización rigurosa de las técnicas de fisioterapia respiratoria; c) Una terapia de comparación apropiada; d) Definir los resultados de interés clínico: mejoría clínica, resolución de los síntomas, signos y resultados de los exámenes de laboratorio, duración de la estancia hospitalaria, duración del tratamiento antibiótico, mortalidad y calidad de vida; y e) Examinar la relación costoefectividad de la terapia.

\section{Bibliografía}

1.- DEPARTAMENTO DE ESTADÍSTICAS E INFORMACIÓN DE SALUD, Ministerio de Salud de Chile. (http://deis.cl/). Revisado el 5 de enero de 2012.

2.- FINE M J, SMITH M A, CARSON C A, MUTHA S S, SANKEY S S, WEISSFELD L A, et al. Prognosis and outcomes of patients with community-acquired pneumonia. A meta-analysis. JAMA 1996; 275: 134-41.

3.- MANDELL L A, WUNDERINK R G, ANZUETO A, BARTLETT J G, CAMPBELL G D, DEAN N C, et al; Infectious Diseases Society of America; American Thoracic Society. Infectious Diseases Society of America/ American Thoracic Society consensus guidelines on the management of community-acquired pneumonia in adults. Clin Infect Dis 2007; 44 (Suppl 2): S27-72.

4.- SOCIEDAD CHILENA DE ENFERMEDADES RESPIRATORIAS Y SOCIEDAD CHILENA DE INFECTOLOGÍA. Manejo de la neumonía del adulto adquirida en la comunidad. Resumen del consenso nacional. Rev Med Chile 2005; 133: 953-67.

5.- LIM W S, BAUDOUIN S V, GEORGE R C, HILL A T, JAMIESON C, LE JEUNE I, et al. Pneumonia Guidelines Committee of the British Thoracic Society Standards of Care Committee. British Thoracic Society guidelines for the management of community acquired pneumonia in adults: update 2009. Thorax 2009; 64 (Suppl 3): 1-55.

6.- MCCOOL F D, ROSEN M J. Nonpharmacologic airway clearance therapies: ACCP evidence-based clinical practice guidelines. Chest 2006; 129 (1 Suppl): 250-9.

7.- BOTT J, BLUMENTHAL S, BUXTON M, ELLUM S, FALCONER C, GARROD R, et al; on behalf of the British Thoracic Society Physiotherapy Guideline Development Group. Guidelines for the physiotherapy management of the adult, medical, spontaneously breathing patient. Thorax 2009; 64 (Suppl 1): i1-i51.

8.- LEVINE A. Chest physical therapy for children with pneumonia. J Am Osteopath Assoc 1978; 78: 122-5.

9.- GRAHAM W G, BRADLEY D A. Efficacy of chest physiotherapy and intermittent positive-pressure breathing in the resolution of pneumonia. N Engl J Med 1978; 299: 624-7.

10.- STAPLETON T. Chest physiotherapy in primary pneumonia. Br Med J (Clin Res Ed) 1985; 291: 143.

11.- BRITTON S, BEJSTEDT M, VEDIN L. Chest physio- 
therapy in primary pneumonia. Br Med J (Clin Res Ed) 1985; 290: 1703-4.

12.- TYDEMAN D. An investigation into the effectiveness of physiotherapy in the treatment of patients with community-acquired pneumonia. Physiotherapy Theory and Practice 1989; 5: 75-81.

13.- BJÖRKQVIST M, WIBERG B, BODIN L, BÁRÁNY M, HOLMBERG H. Bottle-blowing in hospital-treated patients with community-acquired pneumonia. Scand J Infect Dis 1997; 29: 77-82.

14.- NOLL D R, SHORES J, BRYMAN P N, MASTERSON E V. Adjunctive osteopathic manipulative treatment in the elderly hospitalized with pneumonia: a pilot study. J Am Osteopath Assoc 1999; 99: 143-6.

15.- NOLL D R, SHORES J H, GAMBER R G, HERRON K M, SWIFT J Jr. Benefits of osteopathic manipulative treatment for hospitalized elderly patients with pneumonia. J Am Osteopath Assoc 2000; 100: 776-82.

16.- PALUDO C, ZHANG L, LINCHO C S, LEMOS D V, REAL G G, BERGAMIN J A. Chest physical therapy for children hospitalised with acute pneumonia: a randomised controlled trial. Thorax 2008; 63: 791-4.

17.- YANG M, YUPING Y, YIN X, WANG B Y, WU T, LIU G J, DONG B R. Chest physiotherapy for pneumonia in adults. Cochrane Database Syst Rev 2010; (2): CD006338.

18.- SEDOV K R, SMOL'KOVA O V. Value of therapeutic physical culture in the complex treatment of patients with chronic pneumonia. Sovetskaia Meditsina 1975; 1: 65-70.

19.- KUZNETSOV O F. Effectiveness of massage in complex treatment of chronic pneumonia. Voprosy Kurortologii, Fizioterapii i LechebnoiFizicheskoi Kultury 1976; 5: 29-32.

20.- KUZNETSOV O F, LAGUTINA T S. New massage method in the overall treatment of chronic pneumonia. Voprosy Kurortologii, Fizioterapii i Lechebnoi Fizicheskoi Kultury 1980; 3: 13-7.

21.- KUZNETSOV OF, TSAR'KOVA L N, POKROVSKAIA E L, IAKUBSON I M. Effect of massage on the acid-base balance in chronic pneumonia. Voprosy Kurortologii, Fizioterapii i Lechebnoi Fizicheskoi Kultury 1980; 5: 15-20.

22.- VOROB'EV L P, BUSAROVA G A, MERZLIKIN L A, SHESTAKOV V A. Effect of physiotherapy on the outcome of acute pneumonia. Voprosy Kurortologii, Fizioterapii i Lechebnoi Fizicheskoi Kultury 1984; 2: 13-6.

23.- NOLL D. Is osteopathic manipulative treatment (OMT) beneficial for elderly patients hospitalized with pneumonia. www.ClinicalTrials.gov. [: NCT00258661].

24.- FACTO L L. The osteopathic treatment for lobar pneumonia. J Am Osteopathic Assoc 1947; 46: 385-92.

25.- MUNDY L M, LEET T L, DARST K, SCHNITZLER M A, DUNAGAN W C. Early mobilization of patients hospitalized with community-acquired pneumonia. Chest 2003; 124: 883-9.
26.- GILCHRIST F J. Is the use of chest physiotherapy beneficial in children with community-acquired pneumonia? Arch Dis Child 2008; 93: 176-8.

27.- SELSBY D S. Chest physiotherapy. Br Med J 1989; 298: $541-2$.

28.- WALLIS C, PRASAD A. Who needs chest physiotherapy? Moving from anecdote to evidence. Arch Dis Child 1999; 80: 393-7.

29.- GUESSOUS I, CORNUZ J, STOIANOV R, BURNAND B, FITTING J W, YERSIN B, et al. Efficacy of clinical guideline implementation to improve the appropriateness of chest physiotherapy prescription among inpatients with community-acquired pneumonia. Respir Med 2008; 102: 1257-63.

30.- MAIN E, PRASAD A, SCHANS C. Conventional chest physiotherapy compared to other airway clearance techniques for cystic fibrosis. Cochrane Database Syst Rev 2005; (1): CD002011.

31.- ELKINS M R, JONES A, VAN DER SCHANS C. Positive expiratory pressure physiotherapy for airway clearance in people with cystic fibrosis. Cochrane Database Syst Rev 2006; (2): CD003147.

32.- VAN DER SCHANS C P. Conventional chest physical therapy for obstructive lung disease. Respir Care 2007; 52: 1198-206.

33.- BHOWMIK A, CHAHAL K, AUSTIN G, CHAKRAVORTY I. Improving mucociliary clearance in chronic obstructive pulmonary disease. Respir Med 2009; 103 : 496-502.

34.- ROBINSON K A, MCKOY N, SALDANHA I, ODELOLA O A. Active cycle of breathing technique for cystic fibrosis. Cochrane Database Syst Rev 2010; (11): CD007862.

35.- TANG C Y, TAYLOR N F, BLACKSTOCK F C. Chest physiotherapy for patients admitted to hospital with an acute exacerbation of chronic obstructive pulmonary disease (COPD): a systematic review. Physiotherapy 2010; 96: 1-13.

36.- CONNORS A F JR, HAMMON W E, MARTIN R J, ROGERS R M. Chest physical therapy. The immediate effect on oxygenation in acutely ill patients. Chest 1980 ; 78: 559-64.

37.- POELAERT J, LANNOY B, VOGELAERS D, EVERAERT J, DECRUYENAERE J, CAPIAU P, et al. Influence of chest physiotherapy on arterial oxygen saturation. Acta Anaesthesiol Belg 1991; 42: 165-70.

38.- COHEN D, HORIUCHI K, KEMPER M, WEISSMAN C. Modulating effects of propofol on metabolic and cardiopulmonary responses to stressful intensive care unit procedures. Crit Care Med 1996; 24: 612-7.

39.- HORIUCHI K, JORDAN D, COHEN D, KEMPER M $\mathrm{C}$, WEISSMAN C. Insights into the increased oxygen demand during chest physiotherapy. Crit Care Med 1997; 25: 1347-51.

40.- AITKEN M L, VINCENT J M, PIERSON D J. Effects of pulmonary function of oral high frequency oscillation 
in normal and asthmatic subjects. Respir Med 1992; 86: 211-4.

41.- CAMPBELL A H, O'CONNELL J M, WILSON F. The effect of chest physiotherapy upon the $\mathrm{FEV}_{1}$ in chronic bronchitis. Med J Aust 1975; 1: 33-5.

42.- CHALUMEAU M, FOIX-L'HELIAS L, SCHEINMANN P, ZUANI P, GENDREL D, DUCOU-LE-
POINTE H. Rib fractures after chest physiotherapy for bronchiolitis or pneumonia in infants. Pediatr Radiol 2002; 32: 644-7.

43.- SIEMPOS I I, VARDAKAS K Z, KOPTERIDES P, FALAGAS M E. Adjunctive therapies for communityacquired pneumonia: a systematic review. J Antimicrob Chemother 2008; 62: 661-8.

Correspondencia a:

Dr. Fernando Saldías Peñafiel

Departamento de Enfermedades Respiratorias

Pontificia Universidad Católica de Chile.

Teléfonos: (562) 6331541 - (562) 3543242

Fax: (562) 6335255

Marcoleta 350, Santiago, Chile.

E-mail: fsaldias@med.puc.cl 